\title{
Monitoring Volcanoes using Seismic Noise Correlations*
}

\author{
Florent Brenguier (1), Daniel Clarke (2), Yosuke Aoki (3), Nikolai M. Shapiro (2) , Michel \\ Campillo (4), Valérie Ferrazzini (1) \\ 1 Piton de la Fournaise Volcano Observatory, Institut de Physique du Globe de Paris. \\ 2 Institut de Physique du Globe de Paris, Sorbonne Paris Diderot, CNRS (UMR 7154), Paris, France. \\ 3 Earthquake Research Institute, University of Tokyo. \\ 4 Institut de Sciences de la Terre, Université Joseph Fourier, Grenoble.
}

\begin{abstract}
In this paper, we summarize some recent results of measurements of temporal changes of active volcanoes using seismic noise cross-correlations. We first present a novel approach to estimate volcano interior temporal seismic velocity changes. The proposed method allows to measure very small velocity changes $(\approx 0.1 \%)$ with a time resolution as small as one day. The application of that method to Piton de la Fournaise Volcano (La Réunion Island) shows velocity decreases preceding eruptions. Moreover, velocity changes from noise crosscorrelations over 10 years allows to detect transient velocity changes that could be due to long-lasting intrusions of magma without eruptive activity or to pressure buildup associated to the replenishing of the magma reservoir. We also present preliminary results of noise crosscorrelation waveform perturbation associated with the occurrence of dike injection and volcanic eruption. We show that such an analysis leads us to locate the areas of dike injection and eruptive fissures at Piton de la Fournaise Volcano. These recent results suggest that monitoring volcanoes using seismic noise correlations should improve our ability to forecast eruptions, their intensity and thus potential environmental impact.
\end{abstract}

Keywords: Volcano monitoring, Seismic noise, Volcanic eruption forecast

* Surveillance des volcans à partir du bruit de fond sismique

Email address: brenguier@ipgp.fr (Florent Brenguier)

\section{Introduction}

The early detection of volcanic unrest mainly relies on the monitoring of volcanic seismicity and ground deformation. These methods provide insights into the dynamics of magma pressurization and transport. However, despite considerable effort, the precise forecasting of eruptions and their intensity has proven to be difficult. Therefore, there is a constant need for novel observational methods to obtain information about the ongoing volcanic processes. Pressurized volcanic fluids (magma, water) or gas induce deformation and thus perturbations of the elastic properties of volcanic edifices. These small perturbations can be detected as changes of seismic wave properties using repetitive seismic sources (Ratdomopurbo and Poupinet (1995), Grêt et al.(2005), Wegler et al. (2006)). However, none of these approaches were apt to provide a continuous monitoring of volcano elastic properties.

Our work relies on both theoretical and applied results which show that the Green's function between two sensors can be reconstructed from the correlation of seismic noise (see Campillo (2006) for a review). This property has been extensively used to image the Earth's Interior at a global (Nishida et al. (2009)), regional (Shapiro et al. (2005)), or local scale (Brenguier et al. (2007)). In a pioneering work, Sens-Schoenfelder and Wegler (2006) proposed to use the repetitive waveforms of seismic noise cross-correlations to track for subsurface volcanic edifice velocity changes. In this manner, the continuous recording of ambient seismic noise allows continuous monitoring of volcano interiors. 
As in any monitoring technique, the time resolution, fixed by the time between two independent measurements, should be as short as possible. This requirement points to a major limitation of the method of monitoring using seismic noise correlations. More precisely, cross-correlations computed from short time series of ambient seismic noise may not have converged to stable functions. The cross-correlation fluctuations spoil measurements of waveform perturbations linked to volcano interior changes. There are two main origins for residual fluctuations observed for successive noise cross-correlation functions:

- The first is the inadequate intrinsic length of seismic noise record used to converge to a stable cross-correlation function. The length of the seismic noise record required to converge to a stable correlation function is controlled by the nature of the noise, the distance between sensors and by the intrinsic attenuation and scattering properties of the studied medium (Larose et al. (2008)),

- The second is the non-stationarity of ambient seismic noise (e.g., Hadziioannou et al. (2009)). The temporal fluctuations of ambient seismic noise varies depending on the dominant noise sources. These can be seasonal for ocean generated noise sources (e.g., Stehly et al. (2006)) or daily, or weekly for anthropic noise sources (e.g., Bonnefoy-Claudet et al. (2006)).

In the the following, we will describe recent results of temporal monitoring using crosscorrelations of ambient seismic noise at Piton de la Fournaise Volcano. We will present techniques that rely on, 1) the estimate of travel time perturbations of coda waves of noise cross-correlations and 2) the measure of decorrelation of cross-correlation functions. These results suggest that monitoring volcanoes using seismic noise should improve our ability to forecast eruptions, their intensity and thus potential environmental impact.

\section{Piton de la Fournaise Volcano}

Piton de la Fournaise Volcano (PdF) is a hot spot, shield volcano located on La Réunion island in the Indian ocean (Figure 1). It erupted more than 30 times between 2000 and 2010. These eruptions lasted from a few hours to a few months and were associated with the emission of mainly basaltic lava with volume ranging from less than one to tens of million cubic meters (Peltier et al. (2009)). The time period we consider (1999-2008) started and ended with 2 major eruptions, namely the March 1998 eruption (60 million of cubic meter of lava emitted) and the April 2007 eruption associated to the $300 \mathrm{~m}$ high collapse of the main Dolomieu crater (130 million of cubic meter of lava emitted) (Staudacher et al. (2009)). The intense eruptive activity together with a weak tectonic activity makes Piton de la Fournaise Volcano well suited for studies focused on the processes of magma pressurization and injection and for the development of innovative monitoring methods. 


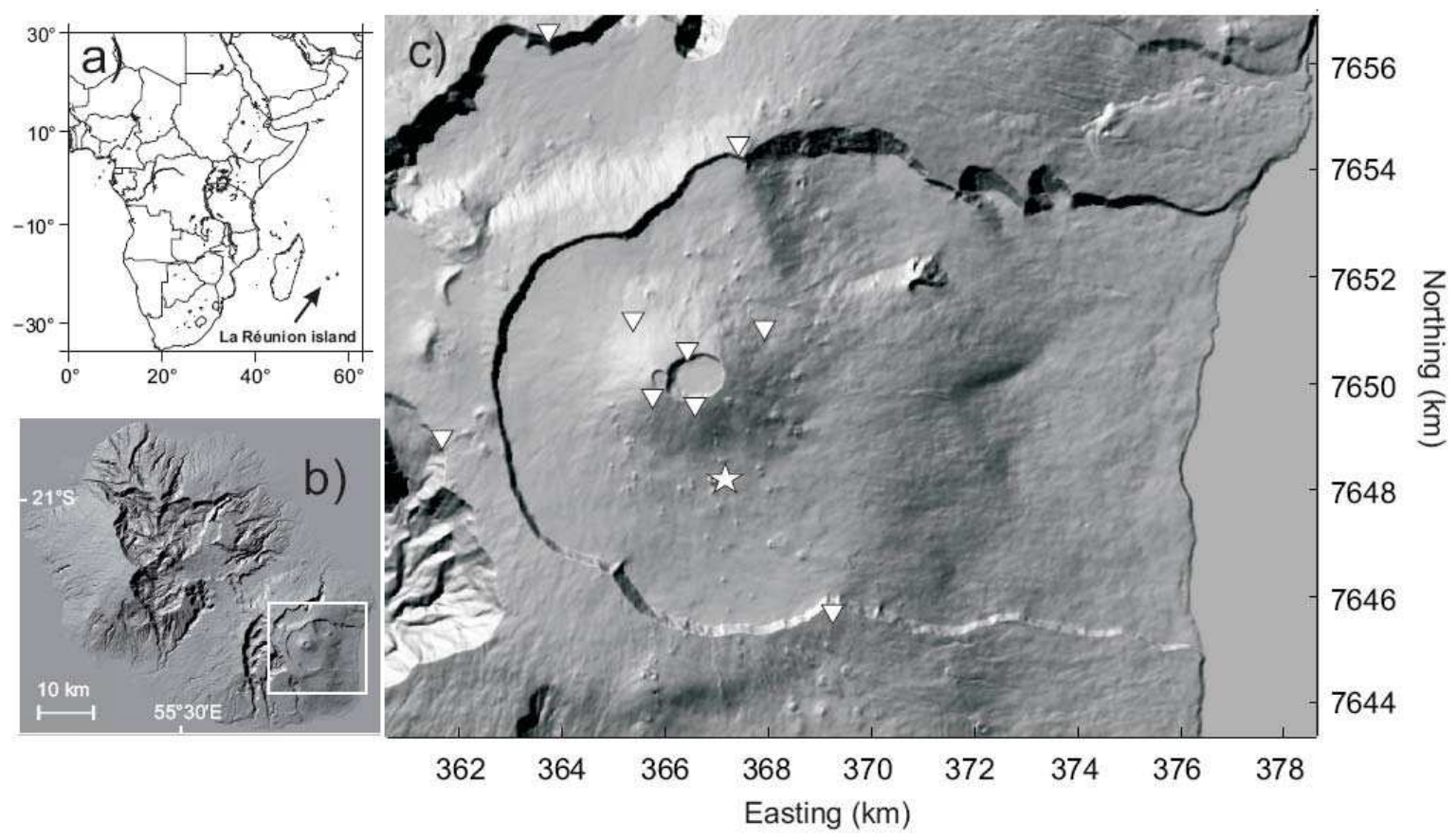

Figure 1: Geographical location of a) La Réunion island, b) Piton de la Fournaise Volcano, and c) the seismic network (inverted triangles) and extensometer (star) used in our analysis.

3. Seismic velocity changes revealed from noise cross-correlations: Application to Piton de la Fournaise Volcano

The method we use consists in measurements of very small waveform time delays in the coda of noise cross-correlations. This method is described as the so-called Moving Window Cross Spectrum (Ratdomopurbo and Poupinet (1995), Clarke et al. (2010)) or Coda Wave Interferometry (Snieder et al. (2002)) techniques. Coda waves (late part of seismograms) are scattered waves that travel long distances and thus accumulate time delays as a consequence, for example, of a uniform seismic velocity change in the propagating medium. Measuring travel time perturbations in the coda thus allows detecting very small velocity changes that would not be detectable by a classical measure of first arrival time delays. The drawback of that approach is that it is difficult to estimate the travel path of the scattered waves constituting the coda. However, recent promising results suggest it may be possible to produce refined 3D maps of small changes in a near future (Larose et al. (2010)).

In a previous work (Brenguier et al. (2008)), we analyzed 18 months (July 1999December 2000) of continuous seismic records from the Piton de la Fournaise (PdF) Volcano Observatory. During the period of study, 5 eruptions occurred lasting on average 26 days each. We applied the Moving Window Cross Spectrum technique between a reference Cross Correlation Function (CCF, stack of 18 months of cross-correlations) and current crosscorrelation functions (ten-day-stacks of CCFs, see Brenguier et al. (2008) for details). As a result, we estimate average (for different receiver pairs) relative travel time shifts $(\Delta \mathrm{T} / \mathrm{T}$ ) which is equivalent to the opposite of the medium uniform relative velocity change $(\Delta \mathrm{V} / \mathrm{V}=-\Delta \mathrm{T} / \mathrm{T})$.

As described by Stehly et al. (2007) and Sens-Schoenfelder (2008), it is important to correct noise records from eventual timing discrepancy because these will perturb the relative travel time shift estimates. Figure 2 a) shows the continuous estimates of relative velocity changes. This curve has been obtained by removing the long-term trend (see Brenguier et al. (2008) for details). This plot clearly shows that the volcanic edifice average seismic velocity decreases by $\approx 0.1 \%$ before each eruption of PdF Volcano. At the same time, 
extensometer data (star on Figure 1 and blue line in Figure 2 a)) only show a precursory opening for the fourth eruption in the series shown in the figure (Peltier et al. (2006)). Figure 2 b) shows inter-eruptive volcano-tectonic seismicity (pre-eruptive swarms are excluded). Seismicity is also clearly a precursor of PdF volcanic eruptions but is more diffuse in time than the precursory velocity changes. We interpret precursory velocity decrease as due to the opening of cracks, which is a consequence of the edifice inflation associated with magma pressurization. A similar observation and interpretation has been proposed by Mordret et al. (2010) for the 2006 eruption of Mt Ruapehu (New-Zealand). Following this approach, Duputel et al. (2009) compared the estimates of seismic velocity changes to deformation recorded by GPS for recent eruptions of PdF Volcano.

Furthermore, to improve the accuracy and thus time resolution of velocity measurements, Baig et al. (2009) developed a cross-correlation filtering method based on time-frequency transforms and phase coherence filtering. This method allowed the detection of a clear decrease in velocity before the June eruption of PdF Volcano (fourth eruption in Figure 2) with a time resolution as small as one day.

Recently, we computed the velocity change time series for 10 years (1999-2008) during which 28 eruptions occurred at PdF Volcano. The entire time series of velocity change will be presented in a joint paper (in preparation). However, as a preliminary result, we observe velocity decreases that are not associated with eruptions (Figure 3 ). These periods are also associated with elevated seismicity. It is interesting to note that these transients last longer (1 or 2 months) than the previously reported pre-eruptive velocity decreases (few days). We thus propose that these transient velocity changes are due to long-lasting intrusions of magma not accompanied by eruptive activity or to pressure buildup associated with the replenishing of the magma reservoir.
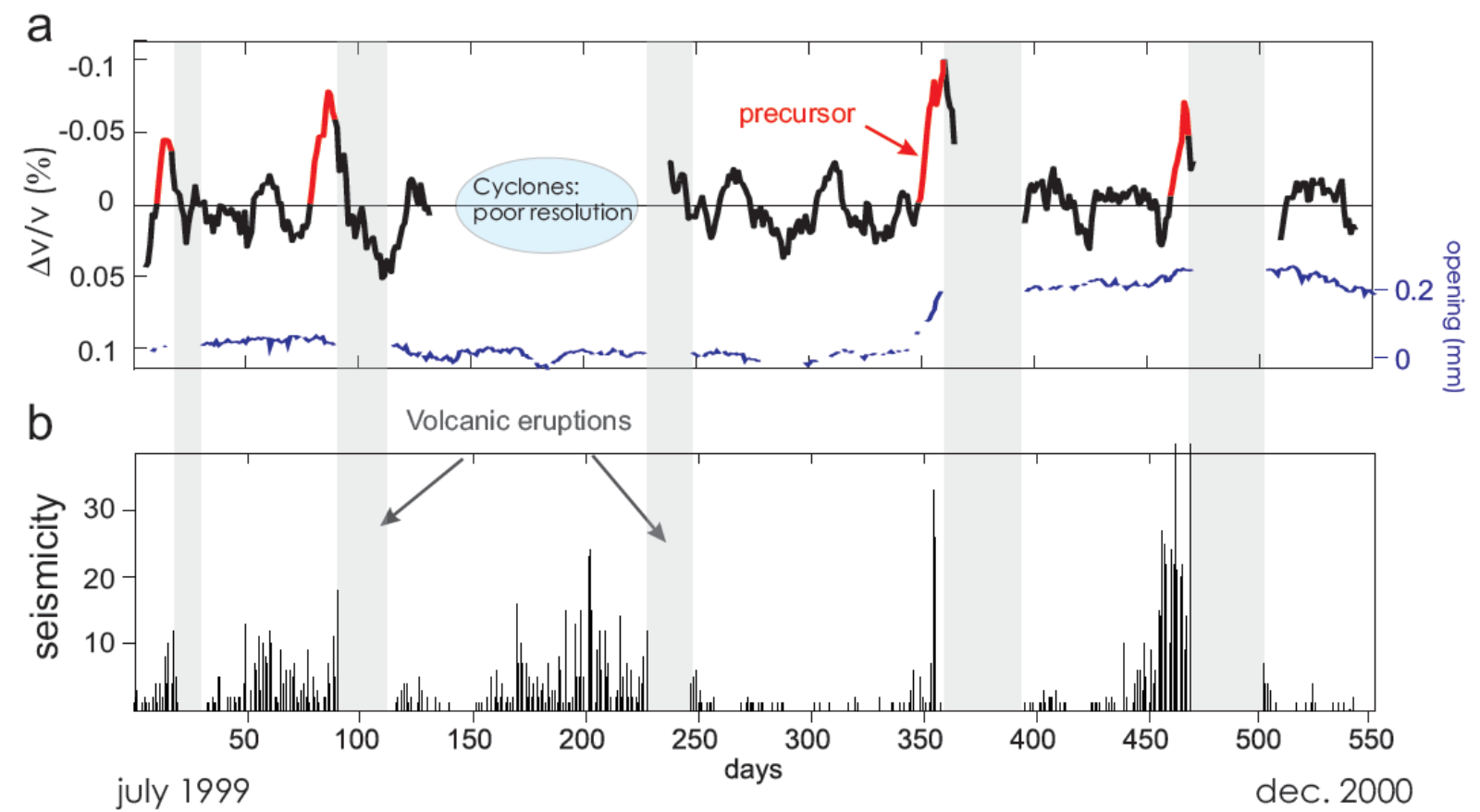

Figure 2: a) Relative velocity changes compared to extensometer (FORX). The travel time shifts are measured in the frequency range 0.1-0.9 Hz. For details, see Brenguier et al. (2008) b) Inter-eruptive seismicity (pre-eruptive swarms are excluded). 

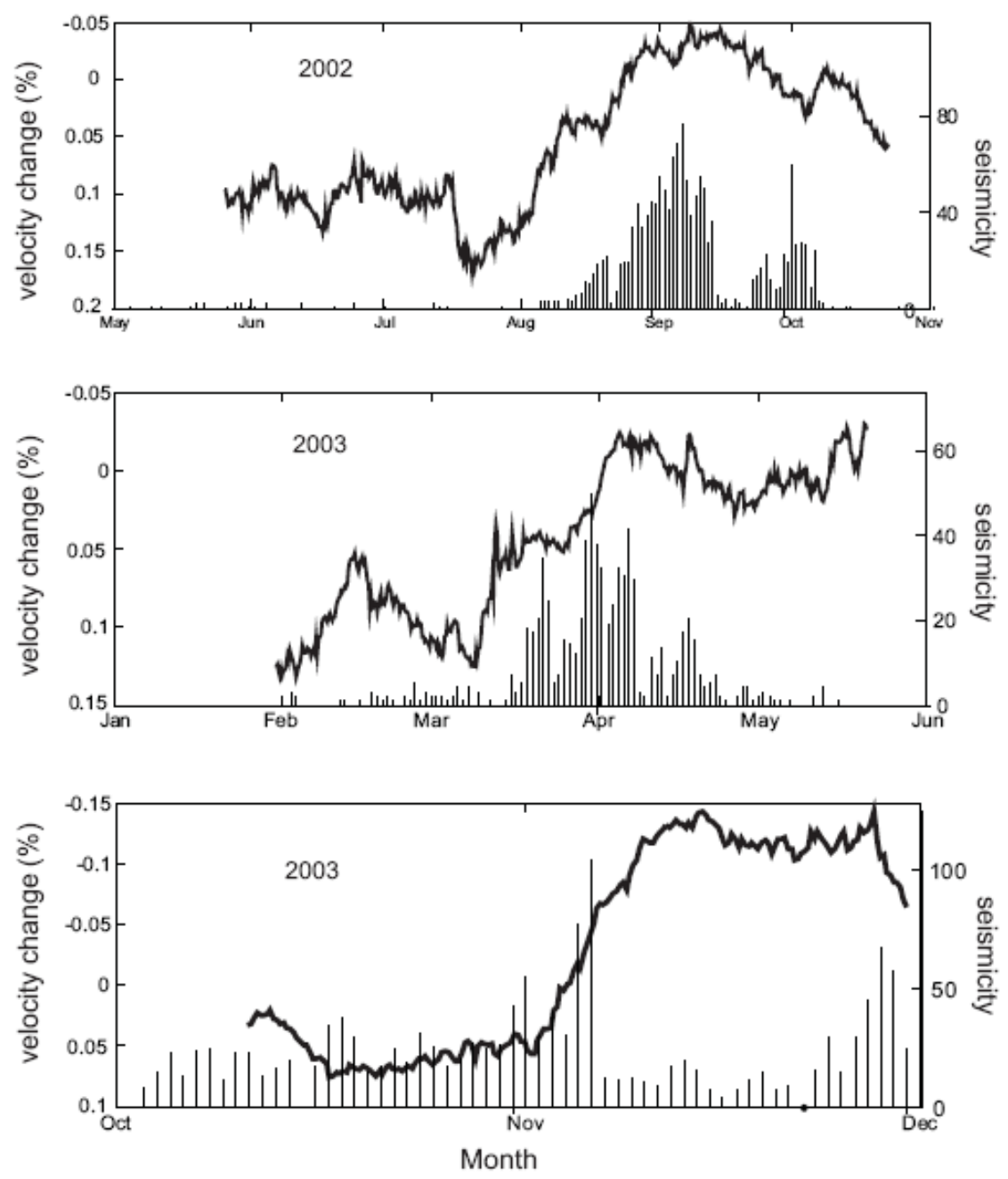

Figure 3: Seismic velocity changes at PdF Volcano not associated with eruptions. Seismicity is given as the number of summit volcano-tectonic events per day excluding seismic swarms.

4. Shallow changes of PdF Volcano detected by decrease of coherence of noise crosscorrelation waveforms

As a complementary approach to the Moving Window Cross Spectrum analysis, the estimate of waveform decorrelation has been used to detect subtle changes within Mount Erebus Volcano (Grêt et al. (2005)), to image the time dependence of single scaterrers on the SanAndreas fault (Taira et al. (2008)), or to locate small changes in a medium (Larose et al. (2010)).

At PdF Volcano, we test the waveform changes of noise Cross-Correlation Functions (CCFs) induced by changes in the near-surface medium properties associated with dike intrusion and volcanic eruptions (Aoki and Ferrazzini (2009)). We focus on the time period between March 29 and April 02 2007. During this period, two eruptions occurred, the first on March 30-31, with duration of 10 hours and location on the South-Eastern flank, and the second on April 2nd located about $8 \mathrm{~km}$ from the summit with duration of 30 days (Figure $4 \mathrm{a}$ ). We compute hourly noise cross-correlation functions for 2 receiver pairs in the frequency range of $0.5-3 \mathrm{~Hz}$ and then average the causal and acausal parts of the signal (Figure 4b). We then compute, for each receiver pair, the correlation coefficients between all possible hourly cross-correlation functions (Figure 4c). Either for the March 30-31 or April 2nd eruption, correlation coefficients computed between before and during these eruptive periods are low 
(Figure $4 \mathrm{~b}$ and $\mathrm{c}$ ). This means that noise cross correlations for receiver pairs fer-NTR and NTR-tkr are significantly perturbed during the March 30-31 and April 2nd eruptions. A possible explanation is that the volcanic tremor signal associated with the eruptions dominates the ambient noise signal and thus strongly perturbs the noise CCFs. Crosscorrelation functions computed between fer and NTR exhibit a strong decorrelation between before and after the March 30-31 eruption, which cannot be explained by a change in the ambient noise properties because NTR-tkr CCFs do not exhibit a similar decorrelation. This is confirmed by the observation that fer-NTR CCFs are stable after the March 30-31 and before the April $2^{\text {nd }}$ eruptions (Figure $4 \mathrm{c}$ ). We thus conclude that the fer-NTR crosscorrelation function decorrelation is caused by the change in the near-surface edifice properties associated with the intrusion of a dike and occurrence of the eruption. This preliminary study shows that monitoring noise cross-correlation waveform decorrelation is a promising tool for locating the areas of magma injection and the eruptive fissures.
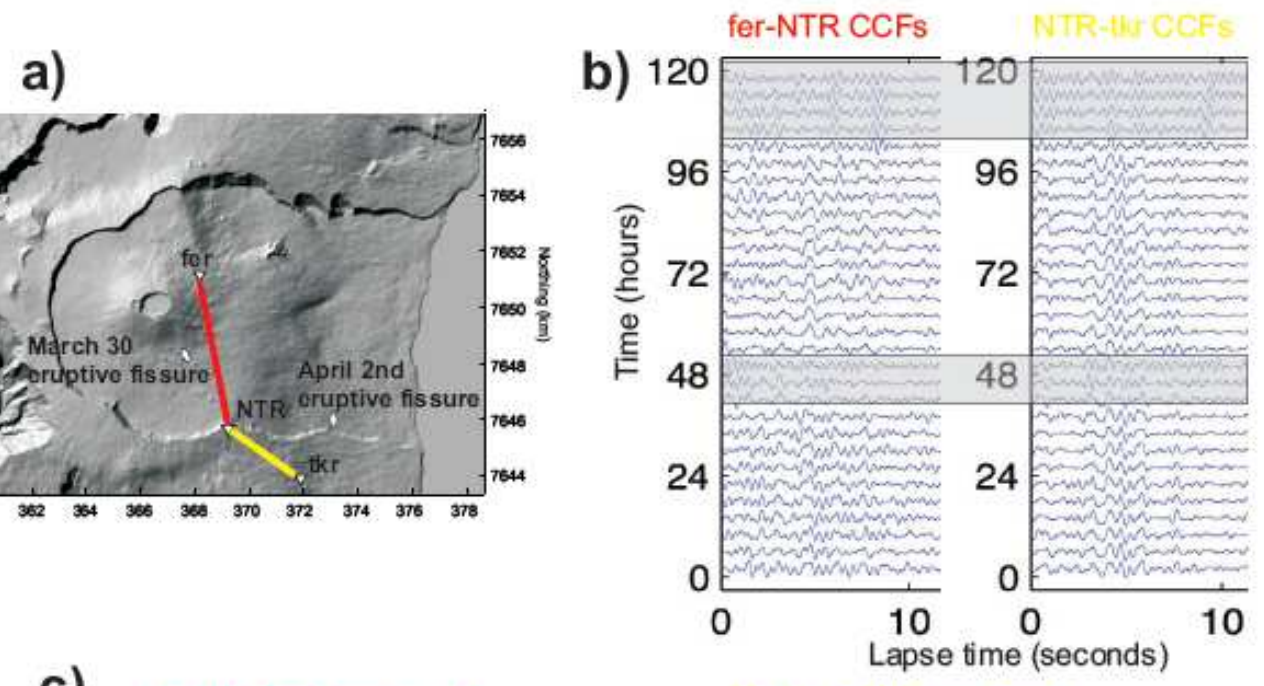

C) fer-NTR CCF corr. coeff.
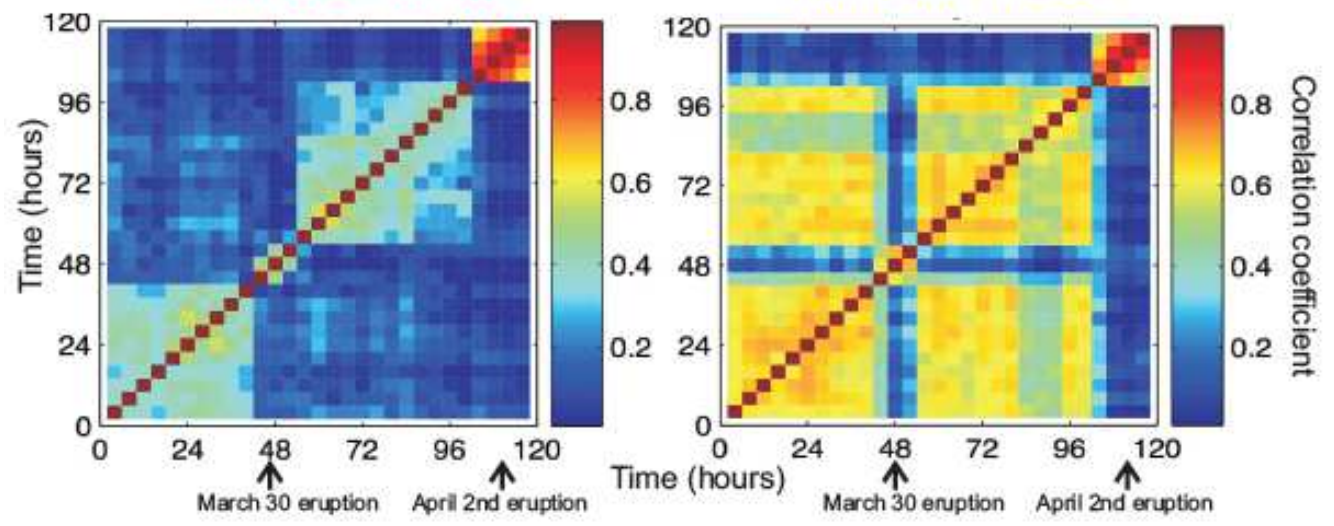

Figure 4: a) Position of March 30 and April 2nd eruptive fissures and of seismic stations used in this study. b) Hourly Cross-Correlation Functions (CCFs). Grey transparent rectangles illustrate periods of eruption c) Correlation coefficients computed between the hourly crosscorrelation functions shown in b). 


\section{Conclusions}

Monitoring the active Earth and in particular volcanoes using seismic noise correlations is a new topic and promising area of investigation. Future methodological challenges concern:

- $\quad$ The reduction of the time resolution. As described previously, filtering techniques using, for example, phase coherence may yield a great improvement,

- The spatial localization of subtle changes detected. This problem will probably require a better understanding of the nature of waves that compose the coda of noise cross-correlations.

Finally it is important to emphasize that these methods are based on the massive processing of continuous seismic records using dense arrays and that future developments will require dense high-quality seismic networks with continuous recording.

\section{Acknowledgments}

All the data used in this study were collected at the Piton de la Fournaise Volcano Observatory. We are grateful to Elodie Rivemale for providing information about intereruptive seismicity, Aline Peltier for providing extensometer data, and Thomas Staudacher for fruitful discussions. We are also grateful to Bernard Chouet for a detailed review of the manuscript. This work has been supported by ANR (France) under contracts 05-CATT-01001 (PRECORSIS), ANR-06-CEXC-005 (COHERSIS), ANR-08-RISK-011 (UNDERVOLC) and by a FP7 European Research Council advanced grant 227507 (WHISPER). This is IPGP contribution number 3105 .

\section{References}

Aoki, Y., Ferrazzini, V., 2009. Temporal changes of seismic wavefield during the 2007 eruptions of Piton de la Fournaise, La Réunion island. In: AGU Fall Meeting Abstracts. p. 03.

Baig, A., Campillo, M., Brenguier, F., 2009. Denoising seismic noise cross correlations. J. Geophys. Res 114.

Bonnefoy-Claudet, S., Cotton, F., Bard, P., 2006. The nature of noise wave field and its applications for site effects studies: A literature review. Earth Science Reviews 79 (3-4), 205227.

Brenguier, F., Shapiro, N., Campillo, M., Ferrazzini, V., Duputel, Z., Coutant, O., Nercessian, A., 2008. Towards forecasting volcanic eruptions using seismic noise. Nature Geoscience 1 (2), 126-130.

Brenguier, F., Shapiro, N., Campillo, M., Nercessian, A., Ferrazzini, V., 2007. 3-D surface wave tomography of the Piton de la Fournaise volcano using seismic noise correlations. Geophysical Research Letters 34 (2), 2305.

Campillo, M., 2006. Phase and Correlation in Random Seismic Fields and the Reconstruction of the Green Function. Pure and Applied Geophysics. 163 (2), 475-502. 
Clarke, D., Zaccarelli, L., Shapiro, N., Brenguier, F., 2010. Monitoring crustal temporal variations from correlations of ambient seismic noise: assessment of resolution and accuracy. Geophysical Journal International in press.

Duputel, Z., Ferrazzini, V., Brenguier, F., Shapiro, N., Campillo, M., Nercessian, A., 2009. Real time monitoring of relative velocity changes using ambient seismic noise at the Piton de la Fournaise volcano (La Réunion) from January 2006 to June 2007. Journal of Volcanology and Geothermal Research 184 (1-2), 164-173.

Grêt, A., Snieder, R., Aster, R., Kyle, P., 2005. Monitoring rapid temporal changes in a volcano with coda wave interferometry. Geophys. Res. Lett., 32, 1-4

Hadziioannou, C., Larose, E., Coutant, O., Roux, P., Campillo, M., 2009. Stability of monitoring weak changes in multiply scattering media with ambient noise correlation: laboratory experiments. The Journal of the Acoustical Society of America 125, 3688.

Larose, E., Planes, T., Rossetto, V., Margerin, L., 2010. Locating a small change in a multiple scattering environment. Applied Physics Letters 96, 204101.

Larose, E., Roux, P., Campillo, M., Derode, A., 2008. Fluctuations of correlations and Green's function reconstruction: role of scattering. Journal of Applied Physics 103 (11), 114907-114907.

Mordret, A., Jolly, A., Duputel, Z., Fournier, N., 2010. Monitoring of phreatic eruptions using Interferometry on Retrieved Cross-Correlation Function from Ambient Seismic Noise: Results from Mt. Ruapehu, New Zealand. Journal of Volcanology and Geothermal Research.

Nishida, K., Montagner, J., Kawakatsu, H., 2009. Global surface wave tomography using seismic hum. Science 326 (5949), 112.

Peltier, A., Bachèlery, P., Staudacher, T., 2009. Magma transport and storage at Piton de La Fournaise (La Réunion) between 1972 and 2007: A review of geophysical and geochemical data. Journal of Volcanology and Geothermal Research 184 (1-2), 93-108.

Peltier, A., Staudacher, T., Catherine, P., Ricard, L., Kowalski, P., Bachèlery, P., 2006. Subtle precursors of volcanic eruptions at Piton de la Fournaise detected by extensometers. Geophys. Res. Lett 33 (6).

Ratdomopurbo, A., Poupinet, G., 1995. Monitoring a temporal change of seismic velocity in a volcano: Application to the 1992 eruption of Mt. Merapi (Indonesia). Geophysical Research Letters 22 (7), 775-778.

Sens-Schoenfelder, C., 2008. Synchronizing seismic networks with ambient noise. Geophysical Journal International 174 (3), 966-970.

Sens-Schoenfelder, C., Wegler, U., 2006. Passive image interferometry and seasonal variations of seismic velocities at Merapi Volcano, Indonesia. Geophys. Res. Lett., 33, 1-5

Shapiro, N., Campillo, M., Stehly, L., Ritzwoller, M., 2005. High-resolution surface-wave tomography from ambient seismic noise. Science 307 (5715), 1615.

Snieder, R., Grêt, A., Douma, H., Scales, J., 2002. Coda wave interferometry for estimating nonlinear behavior in seismic velocity. Science 295 (5563), 2253.

Staudacher, T., Ferrazzini, V., Peltier, A., Kowalski, P., Boissier, P., Catherine, P., Lauret, F., 
Massin, F., 2009. The April 2007 eruption and the Dolomieu crater collapse, two major events at Piton de la Fournaise (La Réunion Island, Indian Ocean). Journal of Volcanology and Geothermale Research 184 (1-2), 126-137.

Stehly, L., Campillo, M., Shapiro, N., 2006. A study of the seismic noise from its long-range correlation properties. J. geophys. Res 111, 1-12.

Stehly, L., Campillo, M., Shapiro, N., 2007. Traveltime measurements from noise correlation: stability and detection of instrumental time-shifts. Geophysical Journal International 171 (1), 223-230.

Taira, T., Silver, P., Niu, F., Nadeau, R., 2008. Detecting seismogenic stress evolution and constraining fault zone rheology in the San Andreas Fault following the 2004 Parkfield earthquake. J. geophys. Res 113, B03303.

Wegler, U., Lühr, B., Snieder, R., Ratdomopurbo, A., 2006. Increase of shear wave velocity before the 1998 eruption of Merapi volcano (Indonesia). Geophys. Res. Lett., 33, 1-4 